\title{
La noción de capacidad de la persona para tomar decisiones, en la práctica médica y legal
}

\author{
G ladys Bórquez E, G ina Raineri $\mathrm{B}^{\mathrm{a}}$, N ina Horwitz $\mathrm{C}^{\mathrm{b}}$, \\ G abriela H uepe $\mathbf{O}^{\mathrm{b}}$.
}

\section{The notion of decision making capacity in medical and legal practice}

Background: The relationship between patients and health professionals emphasizes deliberation and joint decision making, that derives in the informed consent. Aim: To evaluate decision making of patients in health care and to identify the notion of capacity for decision making, according to lawyers and physicians. Material and methods: A semi-structured interview about procedures to assess decision making capacity was applied to 27 selected physicians and lawyers, considering their experience in this area. A qualitative analysis of answers was performed. Results: Several differences were observed between physicians and lawyers, probably originated in their respective disciplines as well as the context of their professional practice. For physicians the notion of capacity is associated to comprehension of the information, it is not absolute, and it must consider the intellectual maturity of the teenager and the autonomy of the elderly. This evaluation is frequently performed in the clinical interview and standardized protocols do not exist. For lawyers, capacity is established by age and is associated to rights and obligations, as determined by law. When it is assessed by experts, including physicians, it becomes evidence. These professionals assume that experts will use standardized assessment instruments. Capacity has significance in the legal system. Conclusions: Since there are substantial consequences when a person is deemed incompetent, it is necessary to distinguish between health capacity and legal capacity, and to link the informed consent with the fundamental rights of citizens, such as taking decisions about our own health (Rev Méd Chile 2007; 135: - ).

(Key words: Decision making; Legislation \& Jurisprudence; Legislation, medical)

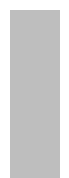

a existencia de una relación diferente entre el Chile.

aAbogada

bocióloga

Recibido el 7 de noviembre, 2006. Aceptado el 20 de marzo, 2007.

${ }^{1}$ Departamento de Bioética y Humanidades Médicas, Facultad de Medicina, Universidad de

Lenfermo y el profesional de la salud, que

Correspondencia a: Gladys Bórquez E. Fax: 56-2-7793850.

Email: gborquez@chp.cl

ARtíCULO DE enfatiza la deliberación y participación conjunta, se traduce en el proceso de consentimiento informado. Así, todo paciente bien informado es capaz de tomar todas las decisiones que lo afectan en su vida y en su salud. En este contexto, una decisión es autónoma cuando cumple tres condi- 
ciones: voluntariedad, información y capacidad, siendo la capacidad la más difícil de evaluarr-3.

La capacidad en salud, definida como la aptitud para desempeñar una tarea específica, en el caso de los pacientes se refiere a tomar decisiones respecto al diagnóstico, tratamiento, pronóstico y cuidado de su enfermedad. De acuerdo con la gravedad de la decisión a tomar, vanían las exigencias de los estándares a medir ${ }^{2-4}$. En la práctica se confunde la capacidad para tomar decisiones en el ámbito médico y legal, lo que es de suma importancia para las personas, toda vez que la consecuencia de ser declarado incapaz, es el impedimento de seguir ejerciendo ese derecho tan personal. Esta tarea, dependiendo del caso al que nos enfrentemos, le es entregada a un tercero, que puede serlo de forma transitoria o definitiva, dependiendo de su situación legal ${ }^{5-17}$.

En una publicación anterior ${ }^{18}$, realizamos una extensa revisión de la reglamentación sanitaria y legal vigente en Chile en torno al tema de la capacidad, con relación a los menores de edad, ancianos y enfermos mentales, marco que ha guiado el planteamiento del presente estudio. La relevancia de investigar empíricamente este problema es crucial, cuando en nuestra sociedad se está revisando la legislación en temas que dicen relación con la sexualidad, la salud, la vida y la muerte.

El presente estudio explora cómo se concibe y evalúa la capacidad de las personas para tomar decisiones en la práctica médica y legal en nuestro país, dando cuenta de los siguientes objetivos específicos: identificar la noción de capacidad para tomar decisiones entre médicos y abogados; identificar los procedimientos utilizados en la práctica médica nacional para estos efectos y conocer cuáles son las diferencias para evaluar la capacidad en los grupos de menores, ancianos y pacientes con problemas de salud mental.

\section{MATERIAL Y MÉTODO}

Para responder a la interrogante básica sobre la valoración y las actitudes hacia la autonomía y la capacidad de las personas, por parte de quienes evalúan esa capacidad, se ha optado por un diseño cualitativo que permite la exploración descriptiva de los elementos centrales en el proceso señalado, documentando cómo se da el fenómeno de manera natural, obteniendo de él la mayor cantidad de información posible sobre los temas más relevantes que intervienen en la construcción mental de los actores $^{19,20}$. No se pretende en esta instancia, por lo tanto, inferir los resultados a nivel nacional.

Instrumento. Se elaboró una entrevista semiestructurada que incluyó dos secciones*. La primera, contiene una serie de preguntas abiertas destinadas a entregar el encuadre para la entrevista y a focalizar la recolección de los datos de interés y la segunda, contiene una escala de diferencial semántico, cuyo propósito es examinar las actitudes y significados asociados a un objeto, en este caso «os procedimientos para evaluar la capacidad». (Los datos recogidos con este segundo instrumento serán objeto de otra publicación). Las entrevistas fueron grabadas previo consentimiento informado de los entrevistados.

Muestra. La unidad de investigación comesponde al profesional: médico en el sistema de salud y abogado en el sistema judicial. El muestreo no es aleatorio, en tanto su propósito es incluir a quiénes y dónde se responden mejor las preguntas del estudio. Se estimó que el punto de saturación de la información**, se alcanzaba con un total de 27 sujetos seleccionados en calidad de informantes claves (Tabla 1).

Análisis de datos. Las entrevistas fueron transcritas y analizadas cualitativamente con la ayuda del programa computacional AQUAD. Se revisó la confiabilidad intercodificador. Para cautelar la calidad de los resultados y asegurar su validez, se recurrió a la triangulación, a través de la intervención de cuatro investigadores, que interpretaron los resultados de manera independiente para posteriormente lograr su consolidación en una discusión interdisciplinaria.

\footnotetext{
* La entrevista puede ser solicitada al autor principal.

* En el diseño cualitativo, la saturación de la información se refiere al momento en la etapa de recolección de datos, en que el investigador evalúa que un caso nuevo ya no añade nada adicional a la información buscada y se puede dar término al trabajo de campo.
} 
Tabla 1

\begin{tabular}{|lll|}
\hline Profesión & Especialidad & $\mathrm{N}^{\circ}$ \\
\hline 14 Médicos & Psiquiatńa Adulto & 4 \\
& Psiquiatńa Infantil & 2 \\
& Neurología Infantil & 3 \\
& Neurología Adulto & 1 \\
& Geriatría & 2 \\
& Adolescencia 1Pediatría/1Ginecología & 2 \\
13 Abogados & Derecho Civil & 1 \\
& Derecho Penal & 2 \\
& Derecho Sanitanio & 3 \\
& Derecho de Familia & 2 \\
& Juez(a) de Menores & 1 \\
& Ministro(a) Corte Apelaciones & 1 \\
& Derecho de Menores & 1 \\
& Notario Público & 2 \\
\hline
\end{tabular}

\section{RESUltados}

Se encontraron interesantes diferencias entre los médicos y abogados estudiados con respecto de cómo se construye el significado y se aplica en la práctica el concepto de capacidad. También se registraron diferencias al comparar a los médicos de distintas especialidades entre sí. A continuación se presentan datos sobre la noción de capacidad y competencia, cómo evaluarlas y las situaciones profesionales en las cuales se evalúa, incorporando las palabras de los sujetos, para resguardar su lógica discursiva en la interpretación.

A. Noción de capacidad y de competencia

A.1. Noción de capacidad en médicos

Entre los médicos se observa un predominio de la noción de capacidad como autonomía, condicionada por el discernimiento del paciente y su comprensión de la información entregada:

«..que sea capaz de poder entender el proceso, de ver las consecuencias a corto y a largo plazo de sus decisiones, que entienda primero qué estamos hablando y segundo que sea capaz de hacerse responsable de alguna manera, de tener responsabilidad frente a sí mismo, respecto a las decisiones que toma...»(Neurólogo Infantil).

Otro importante aspecto asociado a la noción de capacidad es expresado en su calificación de relati- va,» justificada desde dos puntos de vista. Primero, que al hablar de capacidad para tomar decisiones en salud, tanto en el adulto como en el adolescente, la valoración puede ser de capacidad total o parcial. $\mathrm{Si}$ bien se asocia al ejercicio de la autonomía desde el punto de vista legal, referido a la mayoría de edad, se debe hacer una distinción entre la capacidad propia del ámbito del derecho y la capacidad emocional o madurez, más del ámbito médico.

Sin embargo, la noción legal prima muchas veces:

«.. puede que desde un punto de vista emocional tenga la madurez suficiente, pero desde el punto de vista estrictamente médico legal, yo no puedo otorgar capacidad de decisión a un menor de edad...»(Neurólogo Infantil).

En este contexto, establecer la edad como criterio de madurez no sería suficiente:

«ay jóvenes de 18 años super maduros y hay jóvenes de 24 años que son unos cabros chicos...» (Neurólogo Infantil).

La otra razón que justifica el concepto de capacidad «elativa»es la idea de que la capacidad para tomar decisiones en salud no es absoluta, sino que se relaciona con la complejidad de la decisión:

«..generalmente la incapacidad es parcial, porque si uno le dice una cosa de cierta complejidad no va a poder (decidir), pero si a un paciente con cierto grado de demencia y además le duele la cabeza yo le digo ¿quiere tomarse esta aspirina para quitarle el dolor?, él va a ser capaz de decidir sí o no»(Neurólogo).

Lo relativo de la valoración de la capacidad aparece también asociado a transitorio, en el sentido de que pueden existir perturbaciones de la conciencia que regresan:

«..por razones de una enfermedad, como puede ser un traumatismo encéfalo craneal, una depresión muy profunda, una infección o una intervención quirúrgica, momentáneamente la persona puede perder su competencia diaria...» (Geriatra).

Y por último aparece la noción de capacidad asociada con la de autovalencia, la funcionalidad física y psicológica del paciente:

«El geriatra tiene que basar su evaluación en su funcionalidad, o sea su capacidad física que tiene también para llevar a cabo un proyecto de vida»(Geriatra). 
A.2. Noción de capacidad en abogados:

La noción de capacidad en los abogados, en cambio, si bien está también asociada con autonomía, lo que predomina derechamente es el concepto de capacidad legal asociada tanto a mayoría de edad como a deberes y derechos:

«Presunción que hace el Derecho Civil respecto que de las personas de una edad cumplida adquieren tanto el derecho como la obligación....facultad de obligarse y obligar a otros»(Abogado Civilista).

y la definición de incapacidad legal:

Quienes no pueden actuar en la vida jurídica: minoría de edad, enfermedades mentales, sordomudos, pérdida de conciencia y transitorio como ebriedad»(Abogado Penalista).

Sin embargo, también emerge la noción de relatividad:

«i dos pacientes de un psiquiátrico acceden y tienen relaciones sexuales... se estima que sí tienen una capacidad de autodeterminación sexual» (Abogado Penalista).

A.3. Noción de competencia:

Respecto de la noción de competencia y su relación con la noción de capacidad, vemos que para médicos existe confusión:

\&o te diría que fundamentalmente las capacidades podrían usarse como sinónimo...»(Geriatra).

$\varangle$ Yo creo que la competencia tiene que ver con las capacidades técnicas...»(Adolescentóloga).

En cambio para los abogados existe una clara distinción, competencia:

«Facultad que tienen determinados tribunales para fallar dentro de su ámbito de jurisdicción» (Abogado Penalista).

Y capacidad:

«Aptitud que tiene la persona para captar la información que se le está dando para poder valorarla y para poder emitir una decisión....» (Abogada Derecho Sanitario).

\section{B. Cómo se evalúa la capacidad}

\section{B.1. Los Médicos}

\section{- B.1.1. Aspectos Generales}

Entre los médicos existió acuerdo respecto del predominio de la entrevista clínica como herramienta de evaluación, y el reconocimiento de la inexistencia de instrumentos y procedimientos estandarizados y compartidos. La importancia de la entrevista clínica, es que permite el conocimiento del paciente, destacando la evaluación de la capacidad para tomar decisiones como un proceso, más que como un corte en el tiempo; como una tarea integral que involucra a un equipo.

En este sentido, describen además algunos «ndicadores» preferentes de acuerdo a su especialidad médica.

- B.1.1.a. Habilidades de razonamiento, conciencia y discernimiento, lo cual concuerda con la noción de capacidad declarada por los médicos:

«no, si el paciente está sicótico, dos si está deprimido, si de algún modo su enfermedad depresiva le está perturbando su capacidad física y tres, si el paciente tiene algún deterioro intelectual o está con alguna alteración de conciencia»(Psiquiatra).

- B.1.1.b. Capacidad intelectual:

«..la capacidad intelectual de una persona, uno hace pruebas de memoria, pruebas de razonamiento, lingüística y cosas por el estilo...»(Neurólogo).

- B.1.1.c. Capacidad afectiva y emocional:

«ero lo que se evalúa en capacidad fundamentalmente, son capacidades de tipo cognitivo, afectivo y relacional, por lo tanto es una evaluación que habitualmente hacen psicólogos y psiquiatras. Ahora, en general se opera con una evaluación clínica...»(Psiquiatra).

- B.1.1.d. Capacidad de autonomía y autocontrol en caso de los niños:

«..entra un poco la evaluación clínica que hace a través de algún test acerca de la capacidad de autonomía y autocontrol que él tiene sobre sí mismo, supongamos que es un chico con un nivel intelectual limitado, obviamente la parte de la autonomía de él no está como bien desarrollada... pero es diferente en el caso en que el chico tenga un nivel intelectual normal, en que el niño tiene la capacidad de darse cuenta del bien y del mal»(Psiquiatra Infantil).

- B.1.2. Aspectos específicos

- B.1.2.a. En el caso de los especialistas de adolescentes y adultos mayores encontramos una mirada más integral y la utilización de escalas de evaluación:

«odos los que trabajamos con la adolescencia debiera ser igual, digamos una evaluación biopsicosocial... viendo elementos como: cuando se toma una decisión determinada en la información que 
posee acerca del tópico o del tema en que tiene que tomar decisiones...»(Adolescentóloga).

«..escala de evaluación de actividades de vida diaria por ejemplo, para personas que ya tienen alguna discapacidad o déficit, escala de evaluación instrumental para gente que es más autovalente, escala de evaluación para la depresión, escala de evaluación para la función mental o la memoria fundamentalmente, escala de evaluación de la marcha. Escalas psicométricas por ejemplo para evaluar mucho más profundamente lo que es una situación de la evolución cognitiva de las personas»(Geriatra).

- B.1.2.b. Entre los médicos psiquiatras no se observaron coincidencias respecto de los criterios ni de los instrumentos:

«..hablando de la psiquiatría más tradicional en términos de psicosis o en términos de depresión, no existen instrumentos que yo conozca, deben existir a lo mejor en otros lados, pero... yo no conozco instrumentos que sirvan para evaluar capacidad...»(Psiquiatra, Perito).

«..y uno más o menos sigue un punteo en el examen clínico... uno revisa cuál es el aspecto de la persona, cuál es la afectividad, cómo es el lenguaje..., es decir, son aspectos clínicos, digamos»(Psiquiatra Infantil y Perito).

\section{B.2. Los Abogados}

Para los abogados el problema de evaluar la capacidad es una estrategia psicosocial encargada a otros, a expertos, existiendo presunción de la utilización de instrumentos estandarizados que miden discernimiento y conciencia delictiva:

se determina con relación a ciertos informes médicos técnicos, de la asistente social, del psicólogo y otros que señalan su capacidad o incapacidad»(Jueza Menores).

C. Situaciones profesionales en que se evalúa la capacidad

C.1. Situaciones profesionales de médicos:

Las situaciones clínicas que plantean la necesidad de hacer una evaluación son muy frecuentes, parte del trabajo permanente del psiquiatra, neurólogo y adolescentólogo, y relevante para la práctica médica cuando se debe hacer algún procedimiento pero, en su gran mayoría, informales y variadas dependiendo de la especialidad:

«..cuando uno trabaja con gente joven, este fenómeno (de observación) es permanente, porque están en un proceso de crecimiento y de desarrollo y por lo tanto las técnicas nuestras tienen que estar de acuerdo... y ese proceso de crecimiento y desarrollo es parte de la técnica que tiene el profesional en forma automática de estarlo midiendo» (Adolescentólogo).

Para los médicos que atienden niños y jóvenes es donde más se observa una brecha entre la capacidad legal y sanitaria:

«..lo médico-legal es bastante más restringido en general, yo diría que en lo clínico... uno tiene más flexibilidad de acción y de hecho puede incorporar aspectos éticos, humanistas, etc... tampoco en la ley hay una claridad tan... especificada; se define más bien por omisión, o sea, si no existe la condición a, b o c ¿ya?»(Psiquiatra Infantil, Perito).

C.1. Situaciones profesionales de abogados:

Conocer la capacidad de las personas es importante para la práctica profesional de los abogados por sus consecuencias:

«Absolutamente relevante, porque los actos hechos por incapaces son nulos»(Notario).

«Te marca la diferencia entre los que pueden entrar en un caso jurídico y los que no, cómo entra uno y otro, si actúan ellos y son los responsables de sus propios actos o pueden actuar pero autorizados por otros...»(Abogada, Derecho Sanitario).

El ámbito de las situaciones en que se puede plantear la necesidad de evaluar la capacidad puede ser muy amplio, pero su solicitud real es baja, se identifica principalmente el ámbito penal como el espacio de mayor pertinencia:

«En los ámbitos... laboral, para poder trabajar, para poder celebrar un contrato, en el ámbito... menores para saber en qué momento las personas libremente pueden elegir... dejar de estar bajo la tutela de los padres... ámbito penal... cuándo es capaz de cometer un delito y puede ser sancionada, ámbito civil... puede tener patrimonio propio... administrarlo y testar»(Ministro de Corte).

Mayoritariamente no reconocen diferencias entre nociones de capacidad sanitaria y legal, ya que existe presunción de que la Ley incluye las distintas capacidades, además de plantear que no se necesita diferenciarlas. Especialistas reconocen la existencia de cuerpos legales en materias específicas que tratan respecto del derecho de los menores a manifestar su voluntad y otros en materia sanitaria. Si bien no existen diferencias formales en ese sentido, reconocen que en la 
práctica sí existe y que se debería diferenciar la capacidad de las personas en el ámbito sanitario, pero subordinada a la capacidad legal:

«Uponer que una persona por ser menor de edad es incapaz de tomar decisiones en materias sanitarias es una absurda idea»(Juez Menores).

«El término de capacidad en el ámbito legal es muy restringido, por ejemplo es a partir de los 18 años... pero en salud pareciera que la capacidad se entiende en razón de otras cosas» (Abogada Derecho Sanitario).

\section{Discusión}

Se ha recurrido al enfoque cualitativo, suscribiendo el supuesto de que lo social se sustenta en gran medida en lo intersubjetivo y se construye en las actividades, en este caso profesionales, de la vida cotidiana. Es así como describir la estructura básica de esa experiencia -las prácticas habituales de médicos y abogados en la evaluación de la capacidad- busca comprender las acciones significativas para los sujetos y el sentido que éstas adquieren para ellos. Tal sentido está esencialmente ligado a un determinado contexto, donde el mundo social que ahí emerge cobra el carácter ordenado, inteligible $\mathrm{y}$, por lo tanto, familiar de lo que no se cuestiona. En nuestro caso, observamos cómo la primera determinación de las nociones de capaci- dad de los médicos y abogados estudiados, proviene de sus respectivos marcos epistémicos disciplinarios. En segundo término, el contexto y las contingencias de su práctica, se ligan a los matices y diferencias más específicas encontradas, las cuales inspiran mecánicamente acciones de profunda trascendencia ética, como se grafica en la Figura 1.

En nuestro medio, la deliberación en torno a los principios básicos de una bioética plural es aún incipiente. Ella nos exige incluir en la discusión de los fundamentos éticos de nuestras prácticas, la mirada de todos los involucrados ya que no existe «a verdad».

Surge la necesidad de distinguir la capacidad sanitaria de la capacidad legal, específicamente cuando es la mayoría de edad el único criterio para definirla o ante una declaración de interdicción como criterio absoluto, que entrega el poder total al cuidador o tutor legal.

Los médicos que ejercen la geriatría y tienen más claridad de la vinculación que existe entre el consentimiento informado y el ejercicio de derechos de ciudadanía fundamentales, como la gestión del propio cuerpo, es decir, que del resultado de la evaluación de la capacidad de un paciente para tomar decisiones en salud, le puede quedar vedada dicha gestión, siendo entregada a otras personas que la sociedad considera, hasta ahora, especialmente calificadas para ello» médicos, gobernantes, sacerdotes y jueces, lo cual habla del énfasis ético

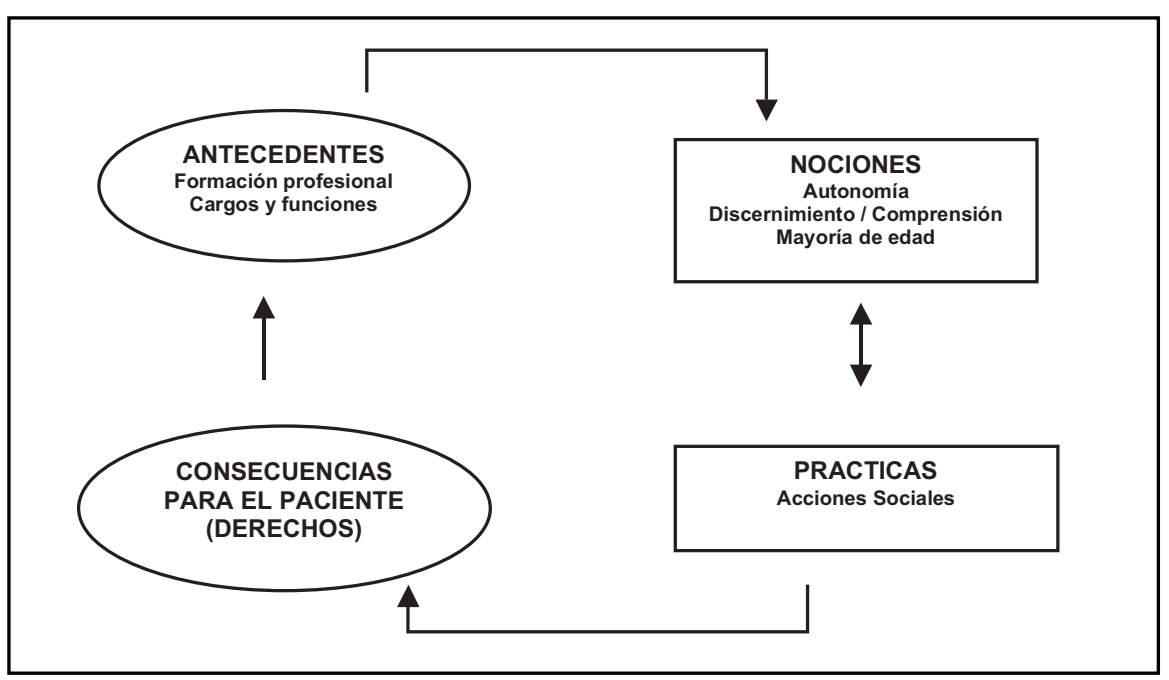

Figura 1. 
que tradicionalmente se ha puesto al principio de beneficencia en la relación médico-paciente.

La información suficiente da validez al consentimiento informado, traducción del respeto a la autonomía de la persona y contribuye a mejorar el acceso equitativo a la asistencia sanitaria, lo que respeta el principio de justicia.

La gran variabilidad observada en la práctica médica de evaluación de la capacidad de los

\section{REFERENCIAS}

1. SÁnchez M. Consentimiento Informado En: Historia y teoría del Método de la Medicina. Introducción al Pensamiento Médico. Barcelona: Ediciones Masson; 1998: 436-437.

2. Simón P. Capacidad o Competencia. En: El Consentimiento Informado. España: Triacastela; 2000.

3. Beauchamp T, Childress J. El Respeto a la Autonomía. En: Principios de Ética Biomédica. Barcelona: Masson S.A.; 1999: 113-172.

4. Drane J. The Many Faces of Competency. Hastings Center Report 1985 (April); 17-21.

5. Buchanan A, Brock D. Deciding for others. The ethics of surrogate decision making. New York: Oxford Univ Press; 1989.

6. Roth L, Meisel A, Lidz C. Test of competency to consent to treatment. Am J Psychiatry 1997; 134: 279-84.

7. Appelbaum P, Roth L. Competency to consent to research A psychiatrics Overview. Arch Gen Psychiatry 1982; 39: 951-58.

8. AleXANDer MP. Clinical Determination of Mental Competence. Arch Neurol 1988; 45: 23-6.

9. Kaplan K, Price M. The Clinican's Role in Competency Evaluations. Gen Hosp Psychiatry 1989; 11: 397-403.

10. FitTen L, Hamann C, Evans GL Relationship of Cognitive Impairment to Decision Making Competence in Nursing Home Residents: Development of a Competence Assessment Protocol. J Am Geriatr Soc 1884; 32 (Suppl): s19.

11. Janofsky JS, MaCarthy JS, Folstein MF. The Hopkins Competency Assessment Test: A brief method for evaluating patient's capacity to give pacientes, puede ser fuente de discriminación y daño para el supuesto incapaz y de falta de protección de un supuesto capaz que no lo es.

Agradecimientos

A la Psicóloga Sra. María de la Luz Bascuñán por sus aportes críticos durante la fase de elaboración del instrumento de medición.

informed consent. Hosp Community Psychiatry 1992; 43: 132-6.

12. Vial del Río V, Lyon A. Derecho Civil, Teoría General de los Actos Jurídicos y de Las Personas. Ediciones Universidad Católica de Chile 1985; 284-6.

13. Código Civil de la República de Chile. Libro IV Título II. De los Actos y Declaración de Voluntad Art. 1445 y siguientes.

14. Romo O. Medicina Legal Elementos de Ciencias Forenses. Editorial Jurídica de Chile 1992; 43-53.

15. Nancuante U, Sotomayor Kiapp R. La Autoridad Sanitaria y Sus principales Potestades. En: Derecho de la Salud. Santiago de Chile Editorial Jurídica Cono Sur Ltda; 2001; 195-20.

16. Maturana A. Código Sanitario. Comentado, Concordado, Jurisprudencia, Leyes y Reglamentos Complementarios. Santiago de Chile: Editorial Jurídica Cono Sur Ltda; Tomo I, 1998 y Tomo II, 1999.

17. Leyes 19.620 sobre Adopción de Menores y 20.084 sobre Responsabilidad de los Adolescentes. Biblioteca del Congreso Nacional de Chile. En http://www.congreso.cl (consultada en octubre 2006).

18. Bórouez G, Raineri G, Bravo M. La evaluación de la "capacidad de la persona": en la práctica actual y en el contexto del consentimiento informado. Rev Méd Chile 2004; 132: 1243-8.

19. Rossman GB, Rawis SF. Learning in the field: An introduction to qualitative research Thousand Oaks, CA: Sage Publications 1998.

20. MAXwELL JA. Qualitative research design. An interactive approach. Thousand Oaks, CA: Sage Publications 1996. 